\title{
Electrical Output Characteristics of Piezoelectric Ceramics Cymbal Transducer with Drop Weight Impact Techniques
}

\author{
Long Wu*, Ming-Cheng Chure, King-Kung Wu, Chia-Cheng Tung \\ Department of Electronics Engineering, Far-East University, Taiwan
}

Copyright (C) 2015 by authors, all rights reserved. Authors agree that this article remains permanently open access under the terms of the Creative Commons Attribution License 4.0 International License

\begin{abstract}
In this study, the relationship between the generated voltages of piezoelectric ceramics Cymbal transducer to which impact mechanical energy was applied was studied. The generated voltages of the piezoelectric ceramics Cymbal transducer increased as the applied mechanical energy were increased. At a single impact mechanical energy, the generated voltages of the piezoelectric ceramics Cymbal transducer were much higher than the voltages of an uncapped piezoelectric ceramics disk. The generated voltages of the piezoelectric ceramics Cymbal transducer depended on the geometric parameters and the metal thickness of the end-cap. The generated voltage of piezoelectric ceramics Cymbal transducer with thick metal end-cap was lower than the voltage of transducers with thin metal end-cap.
\end{abstract}

Keywords Piezoelectric Ceramics, Cymbal Transducer, Energy Harvesting, End-cap

\section{Introduction}

Piezoelectric ceramics can interchange electrical energy and mechanical motion or force. Numerous researchers have studied the power transfer phenomenon exhibited by PZT piezoelectric ceramics. Umeda et al. [1-2] and Xu et al. [3] experimented with free-falling ball to impact a piezoelectric ceramics wafer that was affixed to the under-side, and Umeda et al. [1-2] developed an electrical equivalent model of PZT piezoelectric ceramics that transformed mechanical impact energy into electrical power. Goldfarb et al. [4] presented a linearized model of a PZT piezoelectric ceramics stack and analyzed the efficiency of the stack when it generated power. They determined that the maximal efficiency occurs in a region in which the frequency was much lower than the structural resonance of the stack. Clark and Ramsay [5] compared the poling direction force $\left(\mathrm{d}_{33}\right.$ mode) with the transverse force $\left(\mathrm{d}_{31}\right.$ mode) for use in a PZT piezoelectric ceramics generator, and suggested that the $d_{31}$ mode exhibits a mechanical advantage in converting applied pressure into working stress to generated power. Kasyap et al. [6] created a lumped element model to represent the dynamic behavior of PZT piezoelectric ceramics in multiple energy domains by using an equivalent circuit.

Kim et al. [7-9] used a piezoelectric ceramic Cymbal transducer to harvest energy, the piezoelectric ceramics Cymbal transducer featured two dome-shaped metal end-caps that were affixed to either side of a piezoelectric ceramics circular plate. The magnitude of the piezoelectric energy harvester action was controlled by the product of the effective piezoelectric field constant $d_{\text {eff }}$ and piezoelectric voltage constant geff. The Cymbal transducer exhibited a higher deff - geff than that of other transducer structures such as multilayer stacks and bimorphs. The actual piezoelectric coefficient of the Cymbal transducer was amplified several times because the presence of a cavity enabled the metal end-caps to serve as a mechanical transformer, transforming and amplifying a portion of the incident axial stress in the radial stresses of an opposite sign [7]. Recently, the authors [10] investigated the effect of dimensional size on the output electrical characteristics of uncapped PZT piezoelectric ceramics by using drop weight impact techniques. In this paper, the authors using drop weight impact techniques to study the output electrical characteristics of PZT piezoelectric ceramics Cymbal transducer, and the effects of geometric parameters of metal end-cap on the output electrical characteristics are study also.

\section{Experimental Processes}

The diameter $d_{p}$ and thickness $t_{p}$ of the PZT ceramics disk used in this study were $15 \mathrm{~mm}$ and $0.9 \mathrm{~mm}$, respectively. The PZT piezoelectric ceramics exhibited a free dielectric constant $\varepsilon_{\mathrm{T}}{ }^{33}(1 \mathrm{kHz})$ of 2100 ; a piezoelectric charge constant $\mathrm{d}_{33}$ and $\mathrm{d}_{31}$ of $470 \times 10^{-12} \mathrm{C} / \mathrm{N}$ and $-230 \times 10^{-12} \mathrm{C} / \mathrm{N}$, 
respectively; a piezoelectric voltage constant $\mathrm{g}_{33}$ of $24 \times 10^{-3}$ $\mathrm{Vm} / \mathrm{N}$; a planar electromechanical coupling factor $\mathrm{k}_{\mathrm{p}}$ of 0.70 ; a thickness electromechanical coupling factor $\mathrm{k}_{33}$ of 0.72 ; a mechanical quality factor $\mathrm{Q}_{\mathrm{m}}$ of 65 .

Two types of brass foil were used to create the end-cap of the Cymbal transducer, one with the thickness $t_{b}$ equal to $1 \mathrm{~mm}$, and the other equal to $0.6 \mathrm{~mm}$. The diameter of the end-cap $d_{p}$ was $15 \mathrm{~mm}$, which is equal to the diameter of the PZT ceramics disk; the dimple diameter $\mathrm{d}_{\mathrm{e} 1}$ were $2,3,4$ and $5 \mathrm{~mm}$; the cavity diameter $\mathrm{d}_{\mathrm{e} 2}$ were $5,6,7,8$ and $9 \mathrm{~mm}$; and the cavity depth $t_{\mathrm{e}}$ was $2 \mathrm{~mm}$ (see Fig. 1). Table-1 shows the 12 types of Cymbal transducers with various end-cap parameters that were used in this study.

First, the surfaces of the brass caps flange and the ceramic disk were polished using sand paper in improve contact. Insulating epoxy (Emerson and Cuming) composed of $45 \mathrm{LV}$ epoxy resin and $15 \mathrm{LV}$ resin hardener in a ratio 3:1 was used as a bonding material. After being bonded, the Cymbal flanges were clamped and the epoxy was cured in air for 24 hours at room temperature.

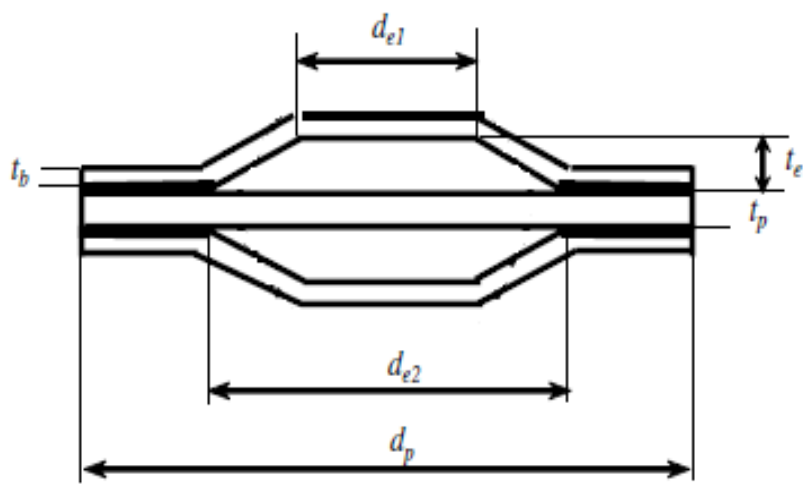

Figure 1. The structure of piezoelectric Cymbal transducer

Table1. The end-cap parameters of the piezoelectric ceramics Cymbal transducers

\begin{tabular}{|c|c|c|c|}
\hline No. & $\begin{array}{c}\text { transducer } \\
\text { diameter, } \mathrm{d}_{\mathrm{p}}(\mathrm{mm})\end{array}$ & $\begin{array}{c}\text { dimple diameter, } \\
\mathrm{d}_{\mathrm{e} 1}(\mathrm{~mm})\end{array}$ & $\begin{array}{c}\text { cavity diameter, } \\
\mathrm{d}_{\mathrm{e} 2}(\mathrm{~mm})\end{array}$ \\
\hline T-1 & 15 & 2 & 9 \\
\hline T-2 & 15 & 3 & 8 \\
\hline T-3 & 15 & 4 & 7 \\
\hline T-4 & 15 & 5 & 6 \\
\hline T-5 & 15 & 2 & 7 \\
\hline T-6 & 15 & 3 & 6 \\
\hline T-7 & 15 & 2 & 5 \\
\hline D-1 & 15 & 3 & 8 \\
\hline D-2 & 15 & 4 & 7 \\
\hline D-3 & 15 & 2 & 7 \\
\hline D-4 & 15 & 3 & 6 \\
\hline D-5 & 15 & 2 & 5 \\
\hline
\end{tabular}

The drop weight impact technique was used to measure the electrical performance of the Cymbal transducer. A steel ball (38.6 $\mathrm{mm}$ in diameter, $16.5 \mathrm{~g}$ in weight) was dropped from a height from 30 to $120 \mathrm{~mm}$, through a steel guide pipe, impacting the test Cymbal transducer. The electrical response of the Cymbal transducer to the applied stress was displayed on a digital storage memory oscilloscope (Agilent MSO-X 3054A) with an input resistance $\mathrm{R}$ of $10^{7} \Omega$; the oscilloscope was connected to a personal computer (PC) for data acquisition and analysis.

\section{Results and Discussions}

Figs. 3 and 4 depict the relationship between the applied mechanical energy and the generated voltage of the Cymbal transducers that had end-caps $1.0 \mathrm{~mm}$ (T-series) and those that had end-caps $0.6 \mathrm{~mm}$ (D-series) in thickness, respectively. The results suggested that regardless of the geometric parameters of the end-cap, the generated voltage of each Cymbal transducer increased as the applied mechanical energy was increased.

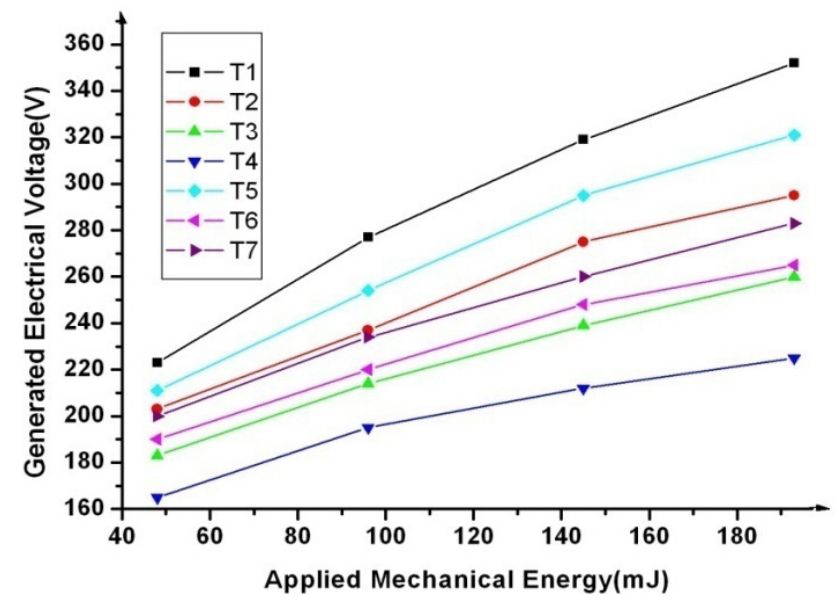

Figure 3. The relationship between applied mechanical energy and generated voltage of T-series Cymbal transducers

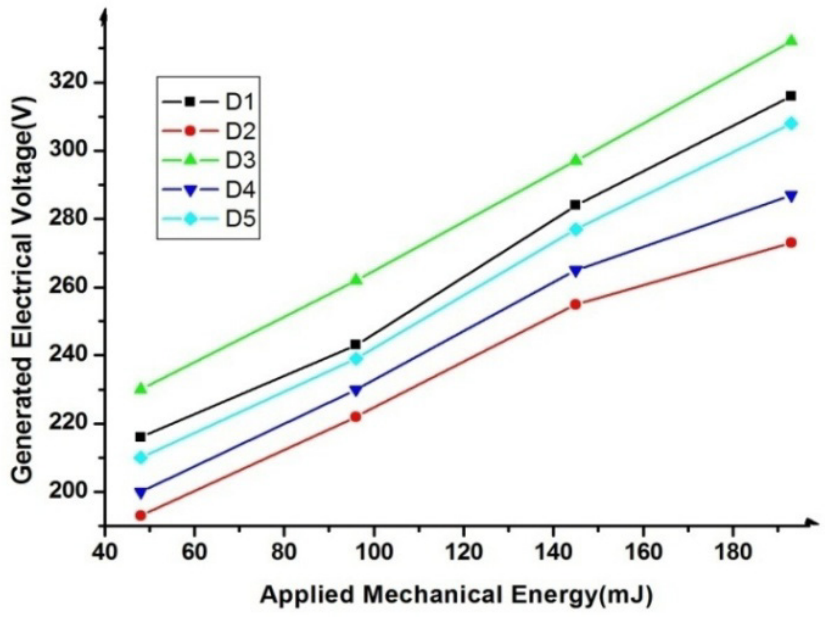

Figure 4. The relationship between applied mechanical energy and generated voltage of D-series Cymbal transducers 
Compared with the results determined in a previous study [10], the results of the present study suggested that the generated voltage of the Cymbal transducer was much higher than that of an uncapped piezoelectric ceramic disk. The results of the previous study [10] indicated that when the applied mechanical energy was $45 \mathrm{~mJ}$, the generated voltage of an uncapped piezoelectric ceramic disk with a diameter of $15 \mathrm{~mm}$ and thickness of $0.9 \mathrm{~mm}$ was approximately $10 \mathrm{~V}$; however, when the same piezoelectric ceramic disk was used as the piezoelectric element in a Cymbal transducer, the generated voltage increased to between approximately $160 \mathrm{~V}$ to $220 \mathrm{~V}$, depending on the end-cap geometry. The amplification factor of the Cymbal transducer used in this study ranged from 16 to 22 .

When the mechanical stress was applied the PZT can generated voltage due to the reverse piezoelectric effect. The relationship between applied mechanical stress and the generated voltage of uncapped PZT piezoelectric ceramics disk can be expressed as [11]:

$$
\mathrm{V}_{\text {un }}=\mathrm{g}_{33} \mathrm{tT}_{3}=\frac{\mathrm{g}_{33} \mathrm{tF}_{3}}{\mathrm{~A}}=\frac{\mathrm{d}_{33}}{\varepsilon_{33}^{\mathrm{T}}} \frac{\mathrm{tF}_{3}}{\mathrm{~A}}
$$

in which $g_{33}$ and $d_{33}$ was the piezoelectric voltage constant and piezoelectric charge constant of the PZT piezoelectric ceramics, respectively; $\varepsilon_{33}^{\mathrm{T}}$ was the free dielectric constant of poled piezoelectric ceramics, A was the surface area of the sample, $\mathrm{t}$ was the thickness of the PZT piezoelectric ceramics, $T_{3}$ was the applied mechanical stress and $F_{3}$ was the applied mechanical force.

The relationship between applied mechanical stress and the generated voltage of piezoelectric ceramics Cymbal transducer can be expressed as:

$$
\mathrm{V}_{\mathrm{Cy}}=\mathrm{g}_{33} \mathrm{tT}_{3}=\frac{\mathrm{g}_{33} \mathrm{tF}_{3}}{\mathrm{~A}}=\frac{\mathrm{d}_{\text {eff }}}{\varepsilon_{33}^{\mathrm{T}}} \frac{\mathrm{tF}_{3}}{\mathrm{~A}}
$$

in which $\mathrm{d}_{\text {eff }}$ was the effective piezoelectric charge constant of piezoelectric ceramics Cymbal transducer caused by the end-cap structure.

The presence of cavities in the piezoelectric ceramics Cymbal transducer allows the metal end-cap to serve as a mechanical transformer for transforming and amplifying a portion of the incident axial stress in the radial stresses of opposite sign [12]. Thus, the $\mathrm{d}_{33}$ and $\mathrm{d}_{31}$ contributions of the PZT piezoelectric disk now combine to yield an effective $d_{\text {eff }}$ value of the piezoelectric ceramics Cymbal transducer as:

$$
\mathrm{d}_{\text {eff }}=\mathrm{d}_{33}+\mathrm{K}\left|\mathrm{d}_{31}\right|
$$

where $\mathrm{K}$ was the amplification factor.
Substitution Eq.(3) into Eq.(2), the generated voltage of piezoelectric ceramics Cymbal transducer can be rewritten as:

$$
\begin{aligned}
\mathrm{V}_{\mathrm{Cy}} & =\frac{\mathrm{d}_{\text {eff }}}{\varepsilon_{33}^{\mathrm{T}}} \frac{\mathrm{tF}_{3}}{\mathrm{~A}}=\left(\mathrm{d}_{33}+\mathrm{K}\left|\mathrm{d}_{31}\right|\right) \frac{\mathrm{tF}_{3}}{\varepsilon_{33}^{\mathrm{T}} \mathrm{A}} \\
& =\frac{\mathrm{d}_{33}}{\varepsilon_{33}^{\mathrm{T}}} \frac{\mathrm{tF}_{3}}{\mathrm{~A}}+\mathrm{K}\left|\mathrm{d}_{31}\right| \frac{\mathrm{tF}_{3}}{\varepsilon_{33}^{\mathrm{T}} \mathrm{A}}=\mathrm{V}_{\text {un }}+\mathrm{K}\left|\mathrm{d}_{31}\right| \frac{\mathrm{tF}_{3}}{\varepsilon_{33}^{\mathrm{T}} \mathrm{A}}
\end{aligned}
$$

From Eq. (4), it found the generated voltage of piezoelectric ceramics Cymbal transducer contain two portions. One portion was caused by the uncapped piezoelectric ceramics disk $V_{u n}$, the other portion was caused by amplify effect of the end-cap cavity. So, with the same applied mechanical stress, the voltage generated by piezoelectric ceramics Cymbal transducer was larger than that generated by uncapped piezoelectric ceramics disk.

Fig. 5 to Fig. 6 shows the relationship between the applied mechanical energy and the generated voltage of Cymbal transducer that had the same dimple diameter $d_{\mathrm{e} 1}$ and different cavity diameter $\mathrm{d}_{\mathrm{e} 2}$. In Fig. 5, the dimple diameter $\mathrm{d}_{\mathrm{e} 1}$ of the transducer was $2 \mathrm{~mm}$, the cavity diameter $\mathrm{d}_{\mathrm{e} 2}$ was $7 \mathrm{~mm}$ for $\mathrm{T} 5$ and $\mathrm{D} 3$ transducers, and the cavity diameter $\mathrm{d}_{\mathrm{e} 2}$ was $5 \mathrm{~mm}$ for T7 and D5 transducers. In Fig. 6, the dimple diameter $\mathrm{d}_{\mathrm{e} 1}$ of the transducer was $3 \mathrm{~mm}$, the cavity diameter $\mathrm{d}_{\mathrm{e} 2}$ was $6 \mathrm{~mm}$ for $\mathrm{T} 6$ and $\mathrm{D} 4$ transducers, and the cavity diameter $\mathrm{d}_{\mathrm{e} 2}$ was $8 \mathrm{~mm}$ for T2 and D1 transducers. The metal thickness of D-series and T-series were $0.6 \mathrm{~mm}$ and $1.0 \mathrm{~mm}$, respectively. The results indicated that, when the dimple diameter $d_{\mathrm{el}}$ was the same; the generated voltage increased as the cavity diameter $\mathrm{d}_{\mathrm{e} 2}$ was increased.

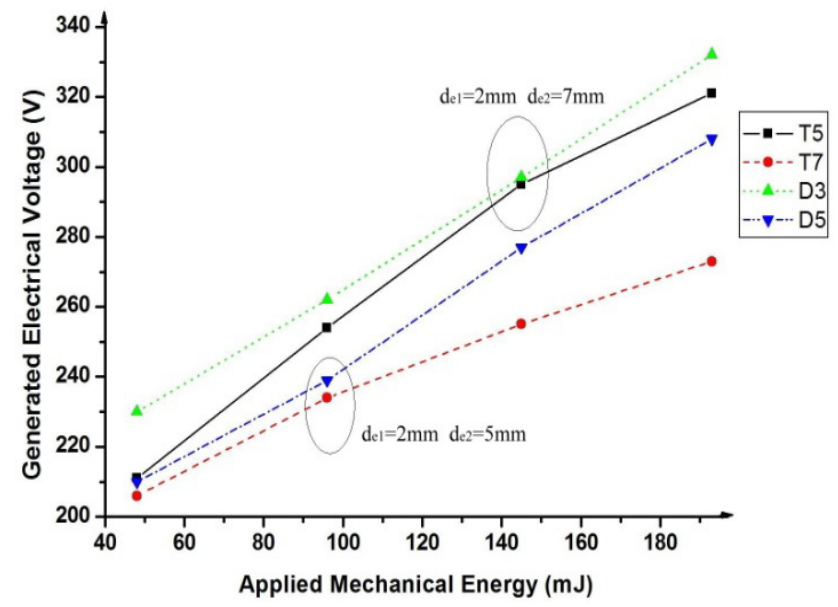

Figure 5. The relationship between applied mechanical energy and generated voltage of T5, T7, D3 and D5 Cymbal transducers 


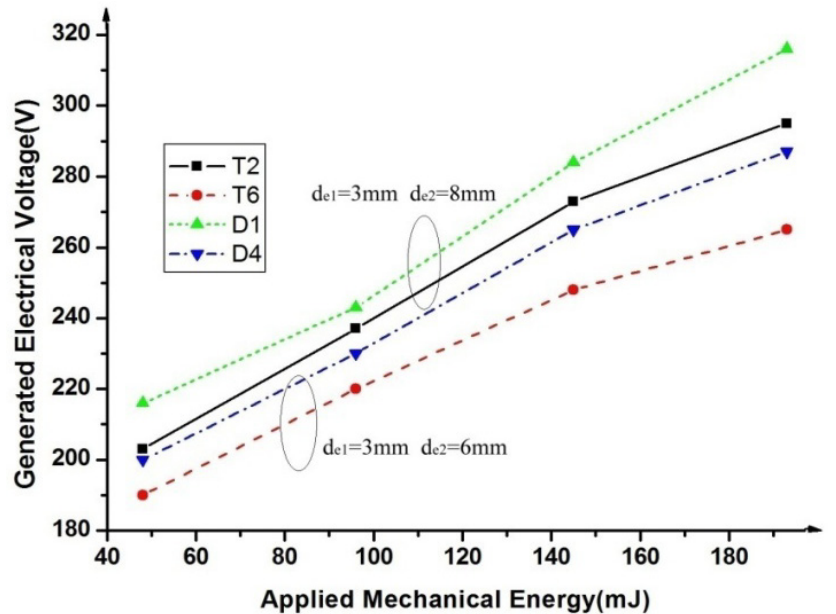

Figure 6. The relationship between applied mechanical energy and generated voltage of T2, T6, D1 and D4 Cymbal transducers

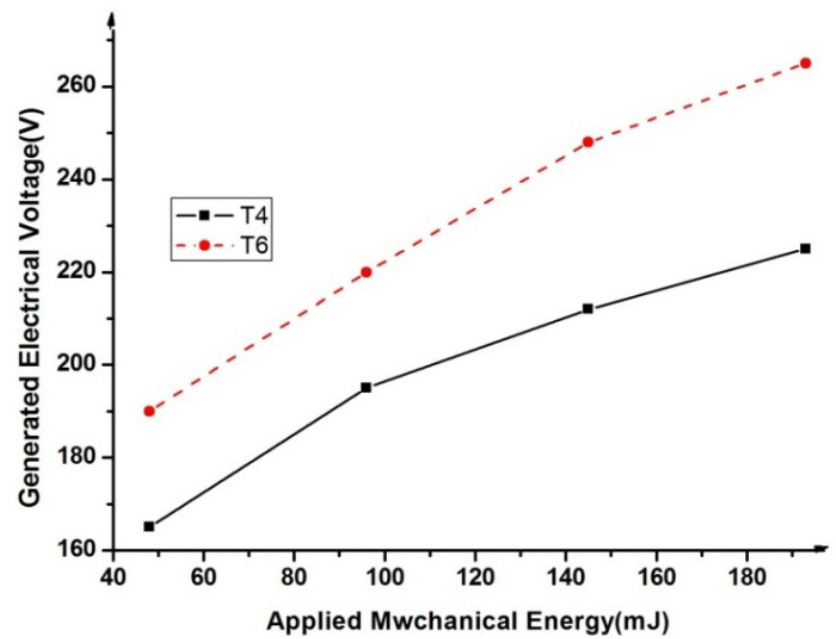

Figure 7. The relationship between applied mechanical energy and generated voltage of T4 and T6 Cymbal transducers

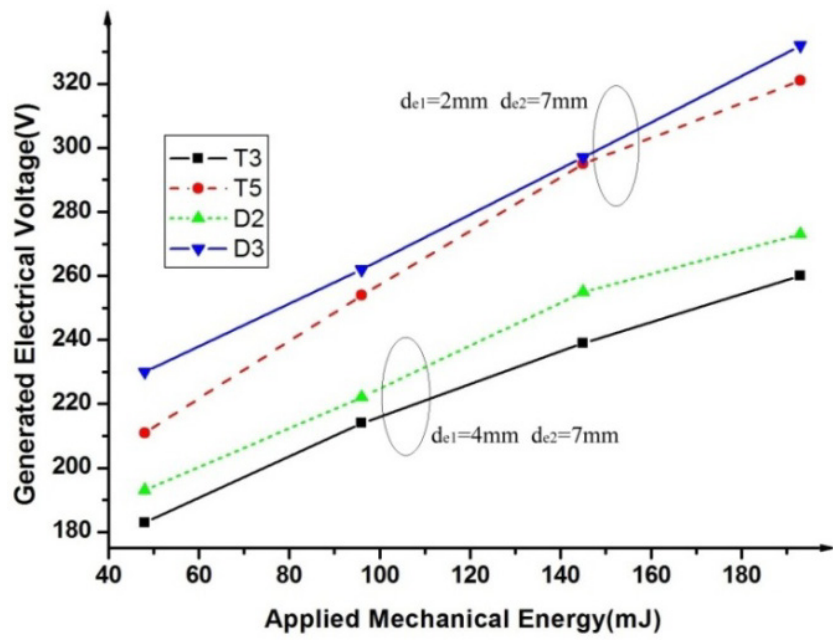

Figure 8. The relationship between applied mechanical energy and generated voltage of T3, T5, D2 and D3 Cymbal transducers

Fig.7 and Fig. 8 illustrate the relationship between the applied mechanical energy and generated voltage of Cymbal transducers that had the same cavity diameter $\mathrm{d}_{\mathrm{e} 2}$ and dissimilar dimple diameter $\mathrm{d}_{\mathrm{e} 1}$. In Fig. 7, the T4 and T6 transducers had a cavity diameter $\mathrm{d}_{\mathrm{e} 2}$ of $6 \mathrm{~mm}$, and a dimple diameter $\mathrm{d}_{\mathrm{e} 1}$ of $5 \mathrm{~mm}$ and $3 \mathrm{~mm}$, respectively. In Fig. 8, the T5 and D3 transducers had the same end-cap geometry with a dimple diameter $\mathrm{d}_{\mathrm{e} 1}$ of $2 \mathrm{~mm}$ and a cavity diameter $\mathrm{d}_{\mathrm{e} 2}$ of $7 \mathrm{~mm}$. The T3 and D2 transducers had the same end-cap geometry with a dimple diameter $\mathrm{d}_{\mathrm{el}}$ of $4 \mathrm{~mm}$ and a cavity diameter $d_{\mathrm{e} 2}$ of $7 \mathrm{~mm}$. The results suggested that, when the cavity diameter $d_{e 2}$ was the same, the generated open circuit voltage decreased as the dimple diameter $\mathrm{d}_{\mathrm{e} 1}$ was reduced.

The amplification factor in Eq.(3) and Eq.(4) depended on the end-cap parameters and can be expressed as [12]:

$$
K=\frac{t_{p}\left(d_{e 2}-d_{e 1}\right)}{t_{e}\left(t_{p}+2 t_{b}\right)}
$$

in which, $t_{p}, t_{e}$ and $t_{b}$ was the thickness of piezoelectric thickness, cavity height and thickness of end-cap metal, respectively. $\mathrm{d}_{\mathrm{e} 1}$ and $\mathrm{d}_{\mathrm{e} 2}$ was the dimple diameter and cavity diameter, respectively.

The effect of end-cap parameters on the generated voltage of piezoelectric ceramics Cymbal transducer can be explain by Eq.(5). From Eq.(5), it found the amplification factor was proportional to the difference between (cavity diameter $\mathrm{d}_{\mathrm{e} 2}$ and dimple diameter $\mathrm{d}_{\mathrm{e} 1}$ ). If the dimple diameter $\mathrm{d}_{\mathrm{e} 1}$ was constant, the difference between (cavity diameter $\mathrm{d}_{\mathrm{e} 2}$ and dimple diameter $\mathrm{d}_{\mathrm{e} 1}$ ) was increased with the increasing of cavity diameter $\mathrm{d}_{\mathrm{e} 2}$, so the amplification factor and also the generated voltage of piezoelectric ceramic Cymbal transducer was increased, as shown in Fig. 5 and Fig. 6. If the cavity diameter $\mathrm{d}_{\mathrm{e} 2}$ was constant, the difference between (cavity diameter $\mathrm{d}_{\mathrm{e} 2}$ and dimple diameter $\mathrm{d}_{\mathrm{e} 1}$ ) was decreased with the increasing of dimple diameter $d_{\mathrm{el}}$, so the amplification factor and also the generated voltage of piezoelectric ceramic Cymbal transducer was decreased, as shown in Fig. 7 and Fig. 8.

Fig. 5, Fig. 6 and Fig. 8 show the relationship between the applied mechanical energy and the generated voltage of transducers with the same end-cap geometry and dissimilar metal thickness. The metal thickness of T-series and D-series samples were $1 \mathrm{~mm}$ and $0.6 \mathrm{~mm}$, respectively. The results implied that, when the same amount of mechanical energy was applied, the generated voltage of the Cymbal transducers with thick metal thickness was lower than that of the transducers with thin metal. The decrease in generated voltage following the increase in metal thickness was attributable to the decrease in $d_{\text {eff }}$ as shown in Eq.(3) to Eq.(5).

\section{Conclusions}

The generated voltage of piezoelectric ceramic Cymbal transducers increased as the applied mechanical energy was increased. At a single impact mechanical energy, the generated voltages of the piezoelectric ceramic Cymbal 
transducer were much higher than the voltages of the uncapped piezoelectric ceramic disk. When the dimple diameter $\mathrm{d}_{\mathrm{e} 1}$ was constant, the generated voltage increased as the increasing of cavity diameter $\mathrm{d}_{\mathrm{e} 2}$. When the cavity diameter $\mathrm{d}_{\mathrm{e} 2}$ was constant, the generated voltage decreased as the dimple diameter $d_{e 1}$ was reduced. The generated voltages of the piezoelectric ceramic Cymbal transducer depended on the metal thickness in addition to the geometry of the end-cap; the generated voltage of piezoelectric ceramic Cymbal transducers with thick metal was lower than that of transducers with thin metal.

\section{REFERENCES}

[1] Umeda M., Nakamura K. and Ueha S. 1996. Analysis of the Transformation of Mechanical Impact to Electrical Energy Using Piezoelectric Vibrator, Jpn. J. Appl. Phys., 35, 3267-3273.

[2] Umeda M., Nakamura K. and Ueha S. 1997. Energy Storage characteristics of A Piezoelectric Generator Using Impact Induces Vibrator, Jpn. J. Appl. Phys., 36, 3146-3151.

[3] Xu C.N., Akiyama M., Nonaka K. and Watanabe T. 1998. Electrical Power Generation Characteristics of PZT Piezoelectric Ceramics, IEEE Trans. Ultrason. Ferroelectr. Freq. Contr., 45(4), 1065-1070.

[4] Goldfarb M. and Jones L.D. 1999. On the Efficiency of Electric Power Generation with Piezoelectric Ceramic, J. Dyn.
Sys. Meas. Control, 121(3), 566-571.

[5] Clark W. and Ramsay M.J. Smart Material Transducers as Power Sources for MEMS Devices, International Symposium on Smart Structures and Microsystems, Hong Kong, 2000.

[6] Kasyap A., Lim J., Johnson D., Horowitz S., Nishida T., Ngo K., Sheplak M. and Cattafesta L. Energy Reclamation from a Vibrating Piezoceramic Composite Beam, Proceedings of 9 th International Congress on Sound and Vibration, Orlando, FL, USA, 2002.

[7] Kim H.W., Batra A., Priya S., Uchino K., Markley D., Newnham R.E. and Hofmann H.F. 2004. Energy Harvesting using a Piezoelectric "Cymbal" Transducer in Dynamic Environment, Jpn. J. Appl. Phys., 43(9), 6178-6183.

[8] Kim H.W., Priya S, Uchino K. and Newnham R.E. 2005. Piezoelectric Energy Harvesting under High Pre-stressed Cyclic Vibrations, J. Electroceram., 15(1), 27-34.

[9] Kim H.W., Priya S. and Uchino K. 2006. Modeling of Piezoelectric Energy Harvesting using Cymbal Transducers, Jpn. J. Appl. Phys., 45(7), 5836-5840.

[10] Chure M.C., Wu L., Wu K.K, Tung C.C., Lin J.S. and Ma W.C. 2014. Power Generation Characteristics of PZT Piezoelectric Ceramics using Drop Weight Impact Techniques: Effect of Dimensional Size, Ceram. Int., 40, 341-345.

[11] Randeraat J.V. and Setterington, R.E. 1974. Piezoelectric Ceramics, Mullard House, London, 1974.

[12] Ochoa P., Villegas M., Pons J. L., Leidinger P. and Fernandez J. F. 2005. Tunability of Cymbals as Piezocomposite Transducers, J. Electroceramics, 14, 221-229. 